\title{
Optical characteristics of intrinsic microcrystalline silicon
}

\author{
Kyung Hoon Jun, Reinhard Carius, and Helmut Stiebig \\ Forschungzentrum Juelich GmbH, Institute for Photovoltaics, D-52425 Juelich, Germany
}

(Received 24 February 2002; published 3 September 2002)

\begin{abstract}
We studied the optical properties of intrinsic microcrystalline silicon $(\mu c-\mathrm{Si}: \mathrm{H})$ deposited by very high frequency plasma-enhanced chemical-vapor deposition system at different silane concentrations (SC) by spectroscopy ellipsometry and photothermal deflection spectroscopy. The bulk property of the samples was probed because the impact of the surface layer was significantly reduced by mechanical polishing. At high SC, we extracted the optical characteristics of the disordered part by assuming a superposition of ideal $c$-Si and mathematically generated amorphous function. At low SC, we studied the reason for a large deviation of absorption coefficient in the energy range between 1.6 and $3.2 \mathrm{eV}$ from the value predicted by effective medium theory. We considered the scattering loss by the inhomogeneity of $\mu c$-Si:H and introduce the dense medium radiative transfer formalism to an optical scattering simulation. We simulated the Stokes vector of $p$ and $s$-polarized light at oblique incidence. From this formalism, we could predict depolarization of $p$ and $s$ wave by scattered incoherent light. Further, we also suggested strain effect as the second possible reason for the enhanced absorption near the onset of the indirect transition in highly crystalline $\mu c-\mathrm{Si}: \mathrm{H}$.
\end{abstract}

DOI: 10.1103/PhysRevB.66.115301

PACS number(s): 78.20.Bh, 78.20.Ci, 78.67.-n

\section{INTRODUCTION}

The recent development of intrinsic microcrystalline silicon $(\mu c-\mathrm{Si}: \mathrm{H})$ enabled the successful incorporation of this material as an active layer in thin-film solar cells. However, the material characteristics remain yet to be better understood. ${ }^{1,2}$ The effect of the microstructure on the optical properties is an important issue for both an in situ characterization of material growth and a clarification of electronic properties. The optical characteristics of structurally inhomogeneous semiconductor such as $\mu c-\mathrm{Si}: \mathrm{H}$ has been interpreted simply as an effective medium of the two-phase (or three-phase) materials ${ }^{3-5}$ although such a microstructure may cause internal light scattering. ${ }^{6,7}$

It is found that the silane concentration SC (gas flow ratio $\left.\left[\mathrm{SiH}_{4}\right] /\left[\mathrm{SiH}_{4}+\mathrm{H}_{2}\right]\right)$ is the most common parameter to control the structure of the material prepared by plasmaenhanced chemical-vapor deposition (PECVD) and the crystalline volume fraction $\left(f_{c}\right)$ decreases with increasing SC. However, near the transition to the amorphous growth regime the highest solar cell efficiencies were achieved. ${ }^{1}$

This paper presents studies of spectroscopic ellipsometry (SE) and photothermal deflections spectroscopy (PDS) of intrinsic $\mu c$-Si:H at different SC. As an approach to separate $\mathrm{H}$ incorporation effect, the results of hydrogenated and dehydrogenated $\mu c$-Si:H samples are compared. Here the viewpoint is adopted that the changes in the band tails of poly-Si can be attributed to disorder that is induced by strain, ${ }^{8}$ as the characteristic exponential tails observed in amorphous silicon $(a-\mathrm{Si}: \mathrm{H})$ are commonly attributed to localized states of strained Si-Si bonds. ${ }^{9}$ In the high-SC regime, we show the optical characteristics of the disordered part in a limited energy range $(1.5 \mathrm{eV}<E<3.2)$ by assuming the optical properties of ideal $c-\mathrm{Si}$. At low $\mathrm{SC}$ special attention is paid to the energy range of $1.6<E<3.2 \mathrm{eV}$, where the absorption coefficient of $\mu c-\mathrm{Si}: \mathrm{H}$ silicon is higher than that for $c-\mathrm{Si}: \mathrm{H}$. The small disordered fraction in a highly crystalline $\mu c-\mathrm{Si}: \mathrm{H}$ cannot account for the large enhancement of the light absorption.

$\mu c-\mathrm{Si}: \mathrm{H}$ or polycrystalline silicon (poly-Si) shows the critical points $(\mathrm{CP})$, near 3.4 and $4.2 \mathrm{eV}$, broadened and shifted to lower energies. Near and above the direct band-gap edge $(\sim 3.4 \mathrm{eV})$, the feature is dominated by direct transitions. ${ }^{10}$ For the $\mathrm{CP}$ in this regime, broadening used to be explained by the scattering at the small grain boundaries imposing a short time for an excited carrier to reach and to be scattered by the grain boundary, thus limiting the excitedstate lifetime. Another major feature in the imaginary part of the dielectric function $\left(\varepsilon_{i}\right)$ occurs clearly near $4.2 \mathrm{eV}$, where an obvious shift of the peak with respect to $c$-Si is observed. ${ }^{10}$ The reason for the deformation of this peak can be more various because the feature is due to several transitions. CP broadening is discussed in more detail by Jellison et al. ${ }^{11}$ (ellipsometry) and Viña and Cardona ${ }^{12}$ (calculation). They have studied the influence of perturbed electronic structure caused by the heavy-doping-effect on the change of the CP's of $\mu c-\mathrm{Si}: \mathrm{H}$.

Further, poly-Si (Ref. 10) or highly crystalline $\mu c-\mathrm{Si}: \mathrm{H}$ (Refs. 2 and 13) has shown a noticeable enhancement of light absorption in the indirect transition regime ( $E$ $<3.4 \mathrm{eV}$ ), however, the reason is not clear yet. To explore the reasons, a scattering loss term is incorporated into the conventional effective medium theory (EMT) and depolarization effects by scattered light are calculated through the dense medium radiative transfer theory. ${ }^{14-16}$ In the past of radiometry, considerable theoretical progress has been made in elucidating and understanding the wave scattering processes by random discrete scatters and rough surfaces. We have simulated the Stokes vector of $p$ - and $s$-polarized light scattered by a slab of homogeneous medium containing spherical scatters using Rayleigh scattering model. ${ }^{14-16}$ However, the dense medium radiative transfer theory needs modification to be applied to semiconductors ${ }^{6,7}$ even though the formalism is derived rigorously. The conventional radiative transfer theory defines the angle of the scattered direc- 
tion for slightly absorptive medium and does not account for coherent wave such as interference from reflection giving rise to interference fringes. To test the applicability of the dense medium radiative transfer equation to the optical simulation for such a nanostructured semiconductor, we took rough modifications of the formalism for a limited energy range. We will show that in the energy range between 1.8 and $3.2 \mathrm{eV}$ the higher absorption in $\mu c-\mathrm{Si}: \mathrm{H}$ in comparison to $c$-Si can be explained by scattering effects at the crystalline grains. However, for energies below $1.8 \mathrm{eV}$ scattering effects are not responsible for the higher absorption. For the higher absorption, instead, we explain by strain. Possible sources for strain will be discussed.

\section{EXPERIMENTS AND MEASUREMENTS}

$\mu c-\mathrm{Si}: \mathrm{H}$ layers were prepared by very high frequency plasma-enhanced chemical-vapor deposition (VHF-PECVD) at $95 \mathrm{MHz}$ from $\mathrm{SiH}_{4}$ and $\mathrm{H}_{2}$ mixtures at different $\mathrm{SC}(2-$ $7 \%$ ), a temperature of $230^{\circ} \mathrm{C}$, a pressure of 0.3 Torr, and a discharge power of $8 \mathrm{~W}\left(\right.$ area $\left.10 \times 10 \mathrm{~cm}^{2}\right)$, yielding deposition rate of $2-5 \AA / \mathrm{sec}^{1}{ }^{1}$ Substrates were Corning 7059 glass for SE and PDS measurements and oxidized $c$-Si for average $\mathrm{H}$ concentration measurements.

In order to study the influence of $\mathrm{H}$ on the optical characteristics of $\mu c-\mathrm{Si}: \mathrm{H}$, we investigated the samples asdeposited and after annealing at $600{ }^{\circ} \mathrm{C}$ for $1 \mathrm{~h}$ in a vacuum chamber (pressure $\sim 2 \times 10^{-6}$ Torr). The annealing temperature was raised gradually for $45 \mathrm{~min}$ and lowered for $3 \mathrm{~h}$.

The average $\mathrm{H}$ concentration of the film before annealing is determined by $\mathrm{H}$ evolution measurements. For average $\mathrm{H}$ concentration measurements, the sample was subsequently annealed in a vacuum furnace (pressure $\sim 2 \times 10^{-6}$ Torr) at temperatures ranging from 200 to $550^{\circ} \mathrm{C}$ for $4 \mathrm{~h}$, in $50^{\circ} \mathrm{C}$ increments from 200 up to $400{ }^{\circ} \mathrm{C}$, and in $25^{\circ} \mathrm{C}$ increments for higher temperatures. Thermal desorption analysis was used to monitor the rate at which $\mathrm{H}$ effuses from a sample while the temperature increased at a constant rate. The experimental setup is described elsewhere. ${ }^{17,18}$

For the characterization of the optical properties in the spectral range between 0.5 and $2.3 \mathrm{eV}$ a conventional PDS setup was used. A typical setup and the evaluation procedure for the rough calculation of the absorption coefficient $(\alpha)$ can be found elsewhere. ${ }^{19-21}$ Here $\alpha$ was calculated from the PDS signal and the synchronously measured transmission signal based on an interferance free formula. ${ }^{21}$ For homogeneous films with known refractive index and thickness, interference free spectra of $\alpha$ are obtained. Inhomogeneities of the optical constants in the growth direction manifest themselves in remaining fringes.

SE measurements were carried out over the spectral range of $1.5-5.0 \mathrm{eV}$ in $0.05-\mathrm{eV}$ steps using a system provided by Jobin Yvon at an angle of incidence $\left(\theta_{0 i}\right)$ of $70^{\circ}$. We analyzed $(\Psi, \Delta)$ spectra, which are defined by

$$
\rho=\frac{R_{p}}{R_{s}}=\tan \Psi e^{i \Delta},
$$

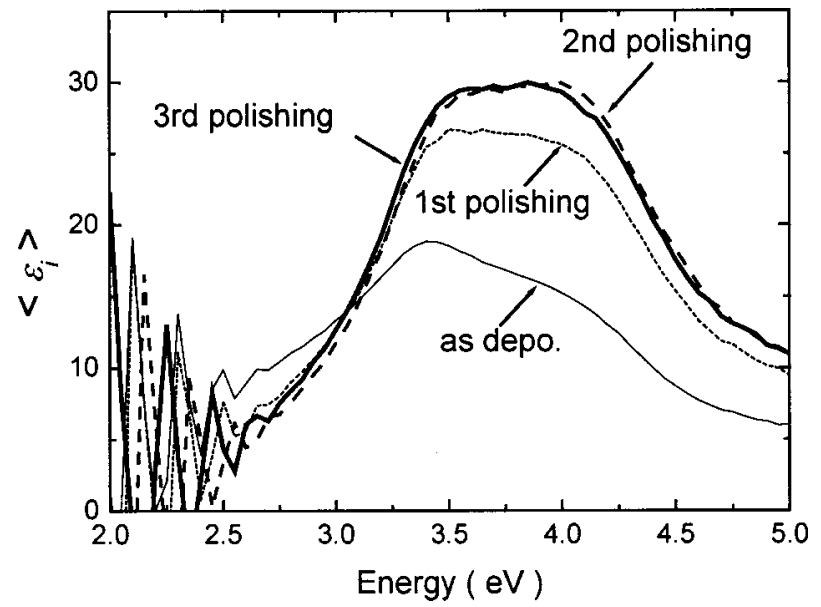

FIG. 1. Imaginary part of the pseudodielectric function $\left\langle\varepsilon_{i}\right\rangle$ of $\mathrm{SC}=4 \% \mu c-\mathrm{Si}: \mathrm{H}$ with an initial thickness of $470 \mathrm{~nm}$. The samples were freshly measured right after each polishing step.

where $\rho$ is the complex amplitude reflection ratio, $R_{p}$ is reflection coefficient of the $p$ wave, and $R_{s}$ is reflection coefficient of the $s$ wave.

To eliminate the surface layers, all the samples were Syton polished on a felt pad, solvent cleaned, and measured iteratively until the ellipsometric response at $E>3.4 \mathrm{eV}$ saturates. As an example, Fig. 1 shows the evolution of the imaginary part of the pseudodielectric function $\left\langle\varepsilon_{i}\right\rangle$ as the surface was polished. The real and imaginary parts of pseudodielectric function, $\left\langle\varepsilon_{r}, \varepsilon_{i}\right\rangle$, of the film/substrate is expressed as follows:

$$
\left\langle\varepsilon_{r}, \varepsilon_{i}\right\rangle=\varepsilon_{0}\left\{\sin \theta_{0 i}\left[1+\left(\frac{1-\rho}{1+\rho}\right)^{2} \tan ^{2} \theta_{0 i}\right]\right\}^{1 / 2} .
$$

After polishing each sample, we determined the remaining root-mean-square roughness $\left(\delta_{\mathrm{rms}}\right)$ of the surfaces by atomic force microscopy (AFM). The AFM measurements were made by a Digital Instruments NanoScope scanning probe microscope in tapping mode with scan window size of 2 $\times 2 \mu \mathrm{m}^{2}$. Values of the polished samples were $\sim 1.5$ $\pm 0.5 \mathrm{~nm}$.

\section{DETERMINATION OF LAYER THICKNESS AND CRYSTALLINE VOLUME FRACTION}

We determined the film thickness, surface roughness, and crystalline volume fraction $\left(f_{c}\right)$ of the samples by modeling $(\Psi, \Delta)$ spectra. Levenberg-Marquardt nonlinear regression algorithm iteratively minimized $\chi^{2}$ in fitting $(\Psi, \Delta)$ data, ${ }^{22}$ where $\chi^{2}$ represents the deviation between the simulated and the measured values. We employed a four-layer stack (air/ surface/bulk/substrate). The surface layer was modeled using the Bruggeman-type ${ }^{23}$ EMT consisting of $50 \%$ voids, and $50 \%$ bulk property. In order to incorporate more degrees of freedom, a fit was attempted using the parametrization of the optical functions of the disordered part from Tauc-Lorentz (TL) equation. ${ }^{24}$ The fit range of the data was limited to the interference part of the spectrum $(1.5-3.2 \mathrm{eV})$, where fairly accurate parameter values can be obtained by assuming stan- 


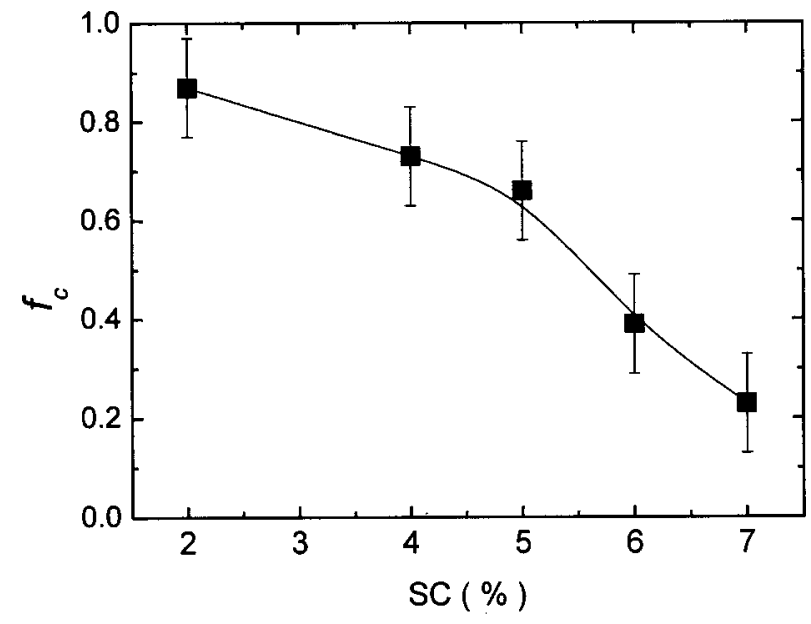

FIG. 2. As-deposited state $f_{c}$ of $\mu c$-Si:H deposited at different SC's determined by modeling of $(\Psi, \Delta)$ spectra. The minimized fitting errors were $\chi^{2}=1.65,0.61,0.31,0.25$, and 0.35 at SC $=2 \%, 4 \%, 5 \%, 6 \%$, and $7 \%$, respectively. We drew a polynomial curve to show the tendency. The error bar indicates the uncertainty of $f_{c}$ by \pm 0.1 , which was deduced by considering the results from the other $f_{c}$ estimation methods.

dard bulk $c$-Si for the crystalline phase. ${ }^{10}$ The surface roughness determined by modeling was $\sim 2.0 \pm 0.5 \mathrm{~nm}$ for all samples, which is in good agreement with the AFM values. This approach cannot cover the high-energy range where the broadened $\mathrm{CP}$ significantly affects the estimation of $f_{c}$. Figure 2 displays the determined $f_{c}$ of the $\mu c$-Si:H deposited at different SC. Houben et al. investigated $n$-doped $\mu c-\mathrm{Si}: \mathrm{H}$ samples from the same deposition system. ${ }^{25}$ The deduced $f_{c}$ from this study is higher than the direct amplitude ratio $c-\mathrm{Si} /(a-\mathrm{Si}+c-\mathrm{Si})$ of the peaks of Raman spectra, ${ }^{25}$ and lower than the estimated value from transmission electron microscopy (TEM) at low $\mathrm{SC}^{25}$ which seems to be the straightforward estimation. In spite of the doping effect in Ref. 25 and difference of the plasma power for deposition (Ref. 25 used $5 \mathrm{~W}$, whereas we used $8 \mathrm{~W}$ ), this trend seems to be valid in the highest crystalline case, because $f_{c}$, from any estimation, saturates to a value when we decrease $\mathrm{SC}$. For example, at $\mathrm{SC}=2 \%, \mathrm{SE}$ determines $f_{c}=0.87$, while amplitude ratio of the Raman peaks indicates 0.79 and TEM estimates over 0.9 . We will discuss the reason why $f_{c}$ by the interpretation of SE data is still lower than TEM estimation in a low-SC regime.

For the next step, the dielectric function $\left(\varepsilon_{r}, \varepsilon_{i}\right)$ of the bulk $\mu c$-Si:H was determined using the thickness of the bulk and the surface, which were obtained by the previous procedure, and solving the ellipsometric equations for $\left(\varepsilon_{r}, \varepsilon_{i}\right)$ of the bulk layer. We used surface roughness value determined by the modeling.

\section{IV. $\mu c$-Si:H WITH LOW- $f_{c}$ CHARACTERISTICS OF AMORPHOUS MATRIX}

This section describes results of $\mu c-\mathrm{Si}: \mathrm{H}$ with low $f_{c}$ $(<0.4)$. In this regime, an extraction of amorphous characteristics from $\mu c-\mathrm{Si}: \mathrm{H}$ by EMT would be hardly affected
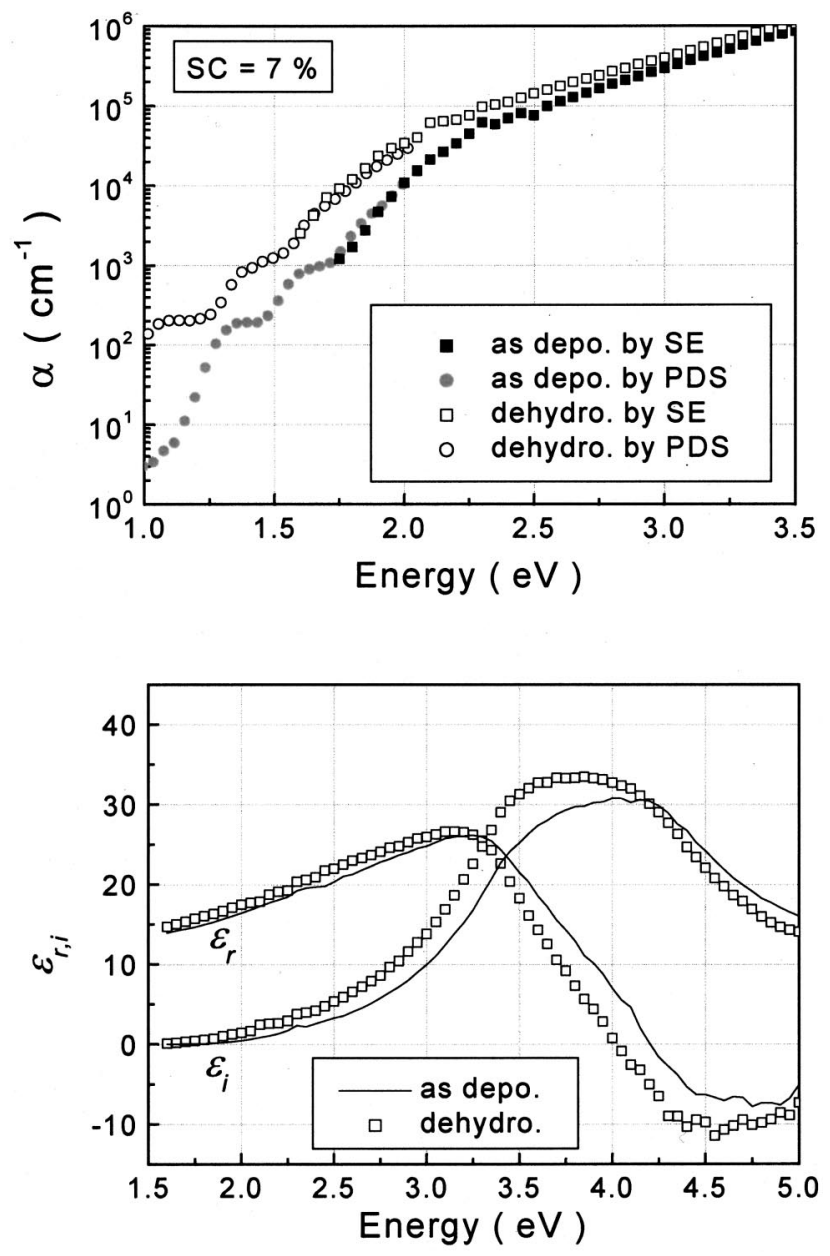

FIG. 3. $\alpha$ and $\left(\varepsilon_{r}, \varepsilon_{i}\right)$ of $\mathrm{SC}=7 \% \mu c-\mathrm{Si}: \mathrm{H}$ in the as-deposited and the dehydrogenated state. $\alpha$ was determined from SE and PDS, and $\left(\varepsilon_{r}, \varepsilon_{i}\right)$ was determined from SE. Some data points scatter due to the numerical instability. We truncated some largely deviating data points in $\alpha$ at the high-energy part of PDS and the low-energy part of SE.

when we assumed crystalline part for the standard bulk value. This section will show that the main feature in this regime is a drastic decrease of $\mathrm{H}$ concentration in $a-\mathrm{Si}: \mathrm{H}$ and development of $c$-Si columnar structure in the film when SC decreases. For simplicity, we adopt the word "H chemical alloy effect" which stands for the change of density of states in amorphous matrix induced by the incorporation of $\mathrm{H}$. A widespread view of the active role of $\mathrm{H}$ is as a homogeneous alloy constituent, ${ }^{26}$ that is, $a$-Si:H might be a $\mathrm{Si}_{1-x} \mathrm{H}_{x}$ alloy with a larger bandgap than pure $a-\mathrm{Si}$.

Figures 3 and 4 display $\alpha$ and $\left(\varepsilon_{r}, \varepsilon_{i}\right)$ of $\mu c-\mathrm{Si}: \mathrm{H}$ in an as-deposited and an annealed state at $\mathrm{SC}=7$ and $6 \%$, respectively. In the energy range between 1.75 and $2 \mathrm{eV}$ we got a good agreement for $\alpha$ using the different measurements indicating the high accuracy of both PDS and SE interpretations. The fringes in the $\mathrm{SC}=7 \%$ sample in the energy range of 1.0-1.8 eV (see Fig. 3) can be attributed to the inhomogeneous growth of the film with higher amorphous volume fraction in the initial growth region than in the bulk. At the onset of crystalline growth condition, the film growth ap- 

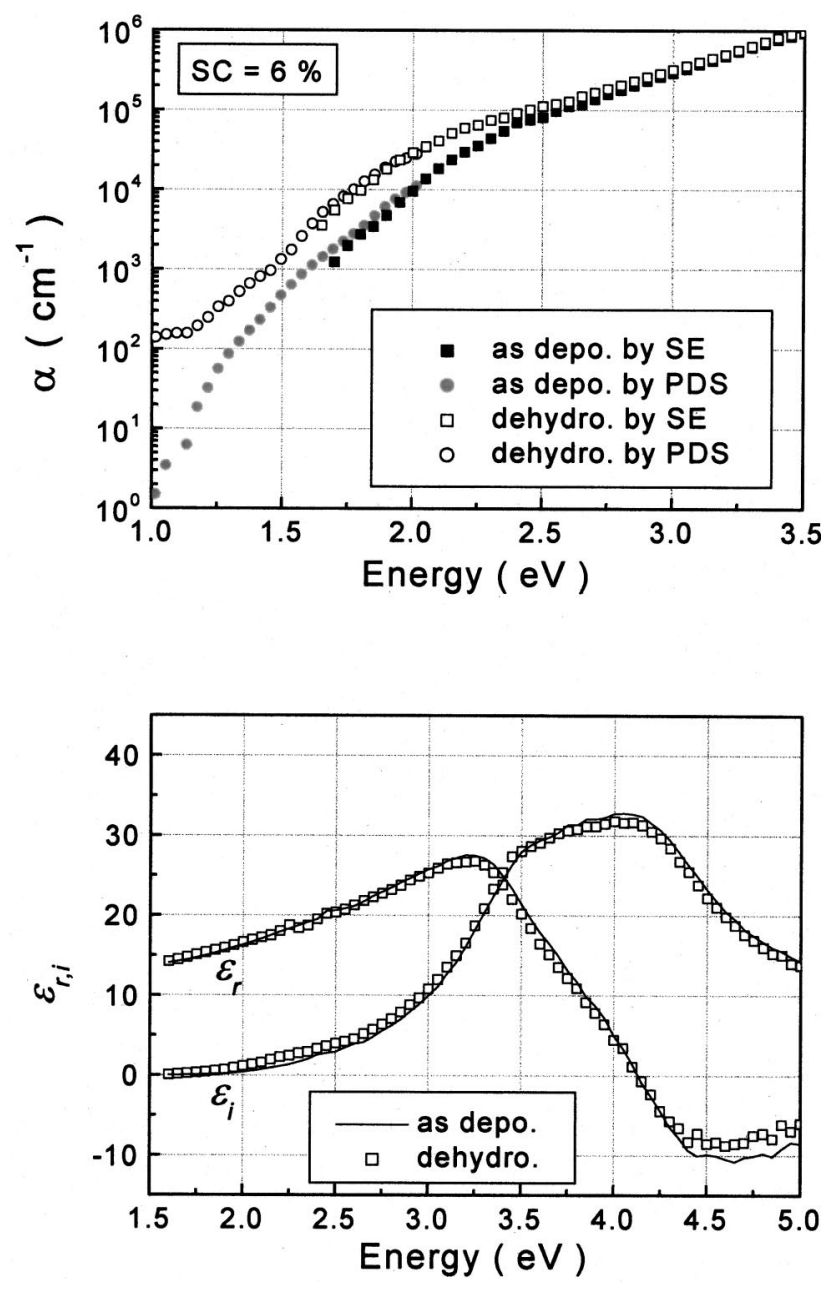

FIG. 4. $\alpha$ and $\left(\varepsilon_{r}, \varepsilon_{i}\right)$ of $\mathrm{SC}=6 \% \mu c-\mathrm{Si}: \mathrm{H}$ in the as-deposited and the dehydrogenated state. $\alpha$ was determined from SE and PDS, and $\left(\varepsilon_{r}, \varepsilon_{i}\right)$ was determined from SE. Some data points scatter due to the numerical instability. We truncated some largely deviating data points in $\alpha$ at the high-energy part of PDS and the low-energy part of SE.

pears predominantly amorphous over an initial layer, that is, there is an incubation layer for the formation of crystalline in contrast with the highly crystalline samples. ${ }^{25}$ Since the film growth is homogeneous, no fringes are observed in Fig. 4. A comparison of both figures allows us to discuss the different optical properties of the films with low $f_{c}$. The main features are as follows.

(1a) The peaks of $\left(\varepsilon_{r}, \varepsilon_{i}\right)$, namely, $\mathrm{CP}$, in $\mathrm{SC}=6 \%$ $\mu c-\mathrm{Si}: \mathrm{H}$ are a bit sharper than $7 \% \mu c-\mathrm{Si}: \mathrm{H}$. For samples with a low $f_{c}$ this feature cannot be attributed to the $\mathrm{CP}$ broadening effect of the crystalline part.

(1b) In the energy range between 1.0 and $3.2 \mathrm{eV},\left(\varepsilon_{r}, \varepsilon_{i}\right)$ and $\alpha$ of both samples are similar in the as-deposited state. That is an unexpected result since $\mathrm{SC}=7 \% \mu c-\mathrm{Si}: \mathrm{H}$ has more $a-\mathrm{Si}: \mathrm{H}$ volume fraction. Generally, $a-\mathrm{Si}: \mathrm{H}$ has a higher amplitude than $c$-Si in this energy range and thus, the SC $=7 \% \mu c-\mathrm{Si}: \mathrm{H}$ should show a higher amplitude of $\left(\varepsilon_{r}, \varepsilon_{i}\right)$ and $\alpha$ than $\mathrm{SC}=6 \% \mu c-\mathrm{Si}: \mathrm{H}$. However, this is not found in these figures.

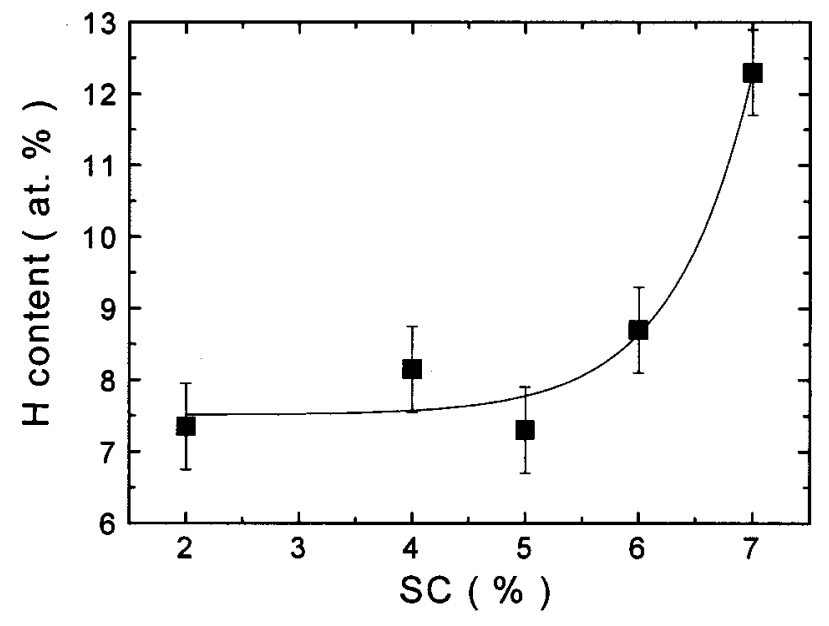

FIG. 5. The average $\mathrm{H}$ concentration in $\mu c$-Si:H films deposited at different SC's. We drew a exponential curve to show the tendency. The error bar indicates \pm 0.5 at $\%$.

(2a) $\left(\varepsilon_{r}, \varepsilon_{i}\right)$ of the dehydrogenated $\mu c-\mathrm{Si}: \mathrm{H}$ at $\mathrm{SC}=7 \%$ changes largely from the as-deposited state. The changes are an enlargement of amplitudes and a strong shift to the lowerenergy side. On the other hand, $\left(\varepsilon_{r}, \varepsilon_{i}\right)$ of the dehydrogenated $\mu c-\mathrm{Si}: \mathrm{H}$ at $\mathrm{SC}=6 \%$ does not change largely from the as-deposited $\mu c-\mathrm{Si}: \mathrm{H}$ as compared to $\mathrm{SC}=7 \% \mu c-\mathrm{Si}: \mathrm{H}$.

(2b) Tails in $\alpha$ in both samples have been increased similarly after dehydrogenation likely due to the increased defects and strained bonds in the amorphous part.

We attribute behaviors (1b) and (2a) to an onset of columnar structure in $\mu c-\mathrm{Si}: \mathrm{H}$ from $\mathrm{SC}=7 \%$ condition and an accompanying reduction of $\mathrm{H}$ concentration in the film. From the TEM investigations, the spherical grain growth in high SC condition have been found to turn into more columnar structure parallel to the growth direction under a decrease in SC. ${ }^{25,27}$ Figure 5 depicts the average $\mathrm{H}$ concentration in $\mu c$-Si:H films at different $\mathrm{SC}$ in an as-deposited state. The $\mathrm{H}$ content increases drastically from 8.5 at. $\%$ at $\mathrm{SC}=6 \%$ to more than 12 at. $\%$ for $\mathrm{SC}=7 \%$. Although $\mathrm{SC}=7 \%$ $\mu c-\mathrm{Si}: \mathrm{H}$ has higher $a-\mathrm{Si}: \mathrm{H}$ volume fraction than $6 \%$ $\mu c-\mathrm{Si}: \mathrm{H}$, the $a-\mathrm{Si}: \mathrm{H}$ network in $\mathrm{SC}=7 \% \mu c-\mathrm{Si}: \mathrm{H}$ has a higher $\mathrm{H}$ content which can be considered as optical microvoids in $a$-Si:H network. When the amplitude of the dielectric function $a-\mathrm{Si}: \mathrm{H}$ is reduced by the amount of incorporated $\mathrm{H}$, the effect of the amplitude enhancement in $\mu c-\mathrm{Si}: \mathrm{H}$ by incorporating $a-\mathrm{Si}: \mathrm{H}$ is not proportional to the amorphous volume faction. As a result, $\alpha$ in the energy range of 1.5-3.2 $\mathrm{eV}$, the response from the amorphous phase dominates, has similar value in both samples, which is the explanation for (1b). In the dehydrogenated state, the $\mathrm{SC}=7 \% \mu c$-Si:H undergoes a strong loss of H-chemical alloy effect, whereas, the change of $\mathrm{H}$-chemical alloy effect is relatively low in SC $=6 \% \mu c$-Si:H. This can account for the difference in peak position shifts described in the first part of (2a). An onset of columnar structure can account for the second part of (2a). The $c$-Si columns in $\mathrm{SC}=7 \% \mu c$ - $\mathrm{Si}: \mathrm{H}$ prevent the thickness reduction after dehydrogenation, the amorphous network in this $\mu c$-Si:H cannot become denser after dehydrogenation. 


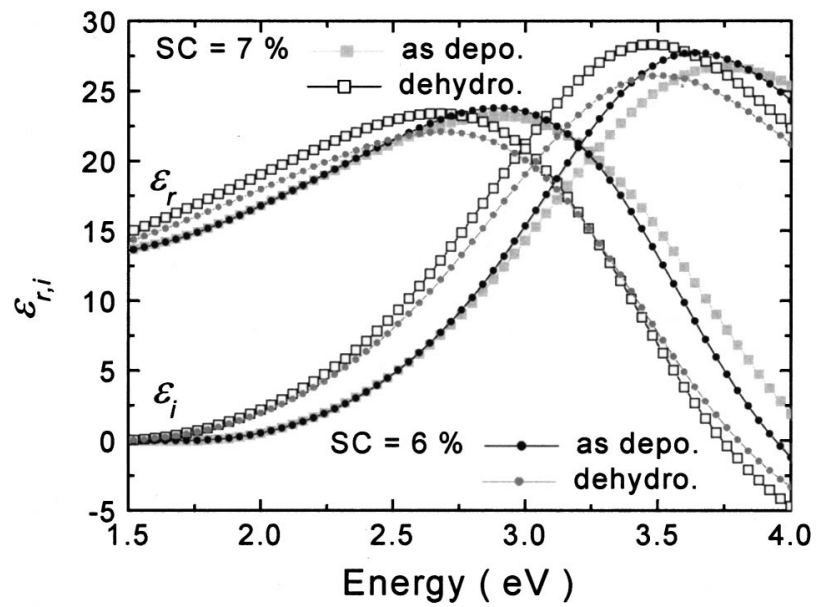

FIG. 6. $\left(\varepsilon_{r}, \varepsilon_{i}\right)$ of the amorphous part in $\mu c-\mathrm{Si}: \mathrm{H}$ in the asdeposited and the dehydrogenated states. One should note that the TL parameters were determined in the energy range from 1.5 to 3.2 $\mathrm{eV}$, however, $\left(\varepsilon_{r}, \varepsilon_{i}\right)$ is shown up to $4.0 \mathrm{eV}$ for clear comparison. For $\mathrm{SC}=7 \% \mu c-\mathrm{Si}: \mathrm{H}, \Gamma=2.09 \mathrm{eV}$ and $E_{0}=3.64 \mathrm{eV}$ in an asdeposited state, and $\Gamma=1.90 \mathrm{eV}$ and $E_{0}=3.42 \mathrm{eV}$ in a dehydrogenated state. For $\mathrm{SC}=6 \% \mu c-\mathrm{Si}: \mathrm{H}, \Gamma=1.79 \mathrm{eV}$ and $E_{0}=3.52 \mathrm{eV}$ in an as-deposited state, and $\Gamma=1.94 \mathrm{eV}$ and $E_{0}=3.45 \mathrm{eV}$ in a dehydrogenated state.

\section{A. Characterization by TL parameters}

As was mentioned, we determined the parameters of TL equation for the amorphous part by fitting in the energy range from 1.5 to $3.2 \mathrm{eV}$, where $\mathrm{CP}$ broadening effect of crystallite can be negligible. We preclude the possible modification of band structure in the crystallites $(1.5<E$ $<3.2 \mathrm{eV}$ ) originating from three-dimensional quantum confinement ${ }^{28}$ due to the large size ( $>20 \mathrm{~nm}$ in diameter) of crystallites in the $\mu c-\mathrm{Si}: \mathrm{H}$ of our study. ${ }^{25}$ Figure 6 shows $\left(\varepsilon_{r}, \varepsilon_{i}\right)$ of the amorphous part of $\mu c-\mathrm{Si}: \mathrm{H}$ in an as-deposited state and a dehydrogenated state. In this case, the "amorphous part" represents contributions from the amorphous matrix, grain boundaries, and voids. We did not assume the void fraction separately in the bulk. Hence, the amorphous part is also representative for the possible void fraction as another free parameter. For clear comparison, we display two TL parameters: (i) oscillator width $(\Gamma)$, which is expected to be inversely proportional to the excited-state lifetime for transitions of electrons and holes deep into the bands of the disordered part; and (ii) peak transition energy $\left(E_{0}\right)$, which indicates the energy position where transition is the strongest, therefore, allows us to discuss the $\mathrm{H}$ chemical-alloy effect by this parameter. For simplicity, we omit the word "amorphous part" in naming of each sample. For $\mathrm{SC}=7 \%$ $\mu c$-Si:H, $\Gamma=2.09 \mathrm{eV}$ and $E_{0}=3.64 \mathrm{eV}$ in the as-deposited state, and $\Gamma=1.90 \mathrm{eV}$ and $E_{0}=3.42 \mathrm{eV}$ in the dehydrogenated state. For $\mathrm{SC}=6 \% \mu c-\mathrm{Si}: \mathrm{H}, \Gamma=1.79 \mathrm{eV}$ and $E_{0}$ $=3.52 \mathrm{eV}$ in the as-deposited state, and $\Gamma=1.94 \mathrm{eV}$ and $E_{0}$ $=3.45 \mathrm{eV}$ in the dehydrogenated state.

(3) In the as-deposited state, the amorphous part in SC $=6 \% \mu c-\mathrm{Si}: \mathrm{H}$ has lower $E_{0}$ and narrower $\Gamma$ than $\mathrm{SC}$ $=7 \% \mu c-\mathrm{Si}: \mathrm{H}$.
(4) Viewing the change of the amorphous part after dehydrogenation, $\mathrm{SC}=7 \% \mu c-\mathrm{Si}: \mathrm{H}$ shows an increase of amplitudes and a decrease of $\Gamma$, and $\mathrm{SC}=6 \% \mu c$-Si:H shows a decrease of amplitudes and an increase of $\Gamma$. Both samples show a strong shift of $E_{0}$ to the lower energy side after dehydrogenation.

The difference of $E_{0}$ in the as-deposited state or strong shift of $E_{0}$ after dehydrogenation can be explained by $\mathrm{H}$ concentration in the film- $\mathrm{H}$ chemical alloying effect, $\mu c-\mathrm{Si}: \mathrm{H}$ with higher $\mathrm{H}$ concentration must show higher $E_{0}$ in its amorphous part. In $\mathrm{SC}=7 \% \mu c-\mathrm{Si}: \mathrm{H}$ case, $\Gamma$ is broader in the as-deposited state than the dehydrogenated state due to local potential fluctuations induced by alloying of $a$-Si with $\mathrm{H}$. In support of this interpretation, one can observe narrower width of $\left(\varepsilon_{r}, \varepsilon_{i}\right)$ of $a$-Si in the dehydrogenated state than the as-deposited state. ${ }^{29}$ In contrast, $\Gamma$ has been increased in $\mathrm{SC}=6 \%$ film after dehydrogenation. We attribute this behavior to an enhanced strain in the amorphous network, occurring from the prevention of thickness reduction after dehydrogenation by the $c$-Si columns. In other words, the surface around $c$-Si column is reconstructed. If the thickness cannot be decreased after dehydrogenation, the amorphous part will undergo a strong strain in its matrix. The decrease of amplitudes in $\mathrm{SC}=6 \% \mu c$ - Si:H after dehydrogenation is attributed to the crystallization of the amorphous part. We found that $f_{c}$ is increased from 0.39 to 0.58 after dehydrogenation in the sample. If the columnar structure prevents the thickness reduction, the space generated in the course of amorphous matrix transforming into $c$-Si, which is denser than amorphous matrix, cannot be compensated.

\section{V. $\mu c$-Si:H WITH HIGH- $f_{c}$ ABSORPTION ENHANCEMENT}

In this section, we discuss $\mu c$-Si:H with high $f_{c}(>0.7)$. We attribute the large deviation of $\alpha$ from the value predicted by EMT in the energy range of $1.6-3.2 \mathrm{eV}$ to the scattering loss arising from inhomogeneity of the material. This section addresses a way to evaluate the depolarization effect by scattered light through dense medium radiative transfer formalism. Also, strain effect in the crystallite and the grain boundary is suggested as the second reason for the high $\alpha$ especially near the onset of indirect transition. In definition of strain, we exclude the two contributions: (i) external strain, which can be caused by substrate and film processing and handling; and (ii) thermal strain, which arises from the disparity between the thermal coefficients of expansion of the substrate and film. For $\mu c-\mathrm{Si}: \mathrm{H}$, the problem is that, like in $c-\mathrm{Si}$, a variety of defect states in the energy region of the band gap can contribute to the absorption and some fraction of the included amorphous phase will also contribute to the absorption. Furthermore, a loss of periodicity in the crystalline phase can be the main obstacle in interpreting measured optical phenomena due to the fact that it will show more or less directlike transition.

Figures 7 and 8 display $\alpha$ and $\left(\varepsilon_{r}, \varepsilon_{i}\right)$ of $\mu c-\mathrm{Si}: \mathrm{H}$ at SC $=4 \%$ and $2 \%$, respectively. We do not show the results of 

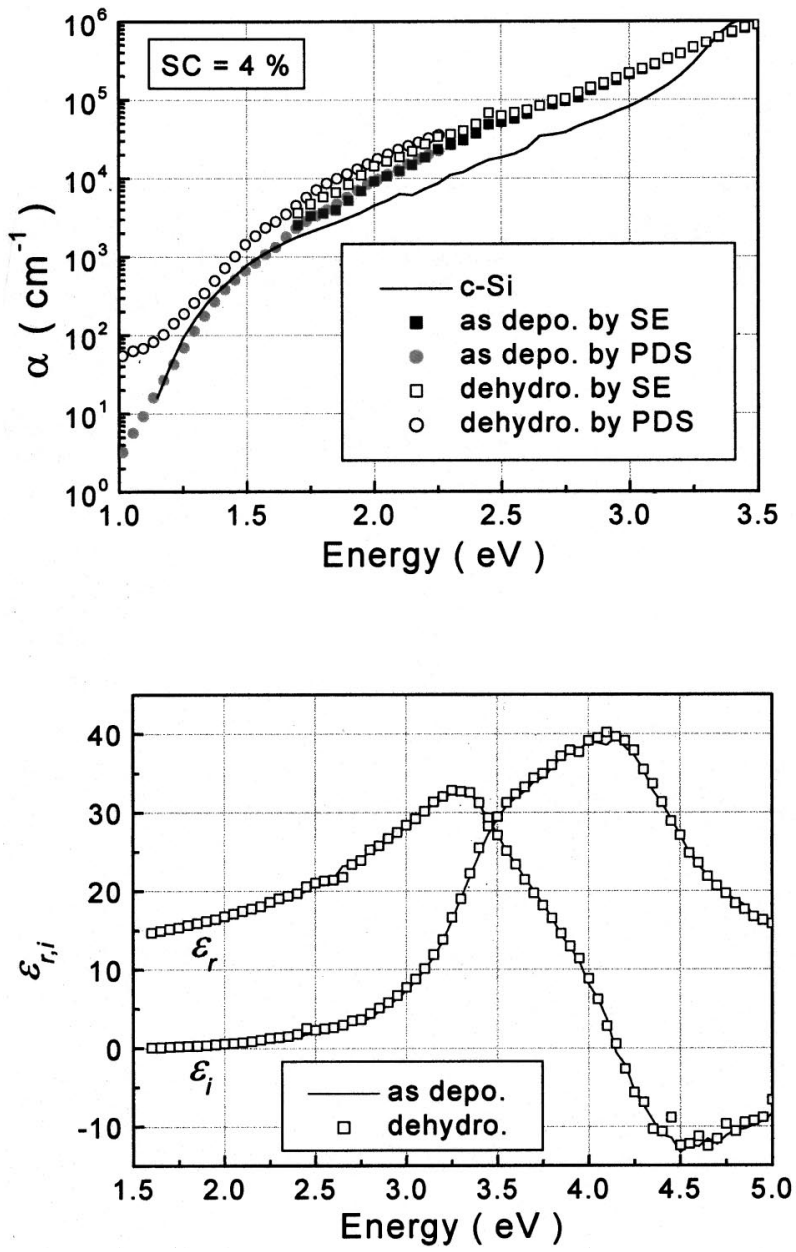

FIG. 7. $\alpha$ and $\left(\varepsilon_{r}, \varepsilon_{i}\right)$ of the $\mathrm{SC}=4 \% \quad \mu c-\mathrm{Si}: \mathrm{H}$ in the asdeposited and the dehydrogenated state. $\alpha$ was determined from SE and PDS, and $\left(\varepsilon_{r}, \varepsilon_{i}\right)$ was determined from SE. Some data points scatter due to the numerical instability. We truncated some largely deviating data points in $\alpha$ at the high-energy part of PDS and the low-energy part of SE. $\alpha$ of $c$-Si is shown as a reference.

$\mathrm{SC}=5 \% \mu c-\mathrm{Si}: \mathrm{H}$ which were similar to those of $\mathrm{SC}$ $=4 \% \mu c-\mathrm{Si}: \mathrm{H}$. We also show $\alpha$ of standard bulk $c$-Si (Ref. 30) as a reference. In both figures, an annealing step has no significant effect on the optical properties of $\mu c-\mathrm{Si}: \mathrm{H}$ with high $f_{c}$. Only in the energy range in the vicinity of the band gap of $c$-Si $(1.0-1.3 \mathrm{eV}) \alpha$ is increased by annealing, which could be attributed to the increased defect density. From the comparison of both figures, we describe the differences in high- $f_{c} \mu c$-Si:H from each $\mathrm{SC}$ :

(5a) $\mathrm{CP}$ of $\left(\varepsilon_{r}, \varepsilon_{i}\right)$ of $\mu c-\mathrm{Si}: \mathrm{H}$ remains largely unchanged upon changes in SC. Only above the onset of columnar growth ( $\mathrm{SC}=7-6 \%)$, noticeable changes in $\left(\varepsilon_{r}, \varepsilon_{i}\right)$ were observed. Here, we do not focus on $\mathrm{CP}$ broadening effect which has been studied elsewhere. ${ }^{10,31,32}$ The reason for $(5 \mathrm{a})$ is believed to be a short electron lifetime caused by grain boundary scattering.

(5b) In both samples, $\alpha$ is excessively higher than the value predicted by EMT in the range $1.6<E<3.2 \mathrm{eV}$.

(5c) $\alpha$ is still high in $1.2<E<1.8 \mathrm{eV}$ where the incorporated amorphous phase, on the contrary, is expected to re-
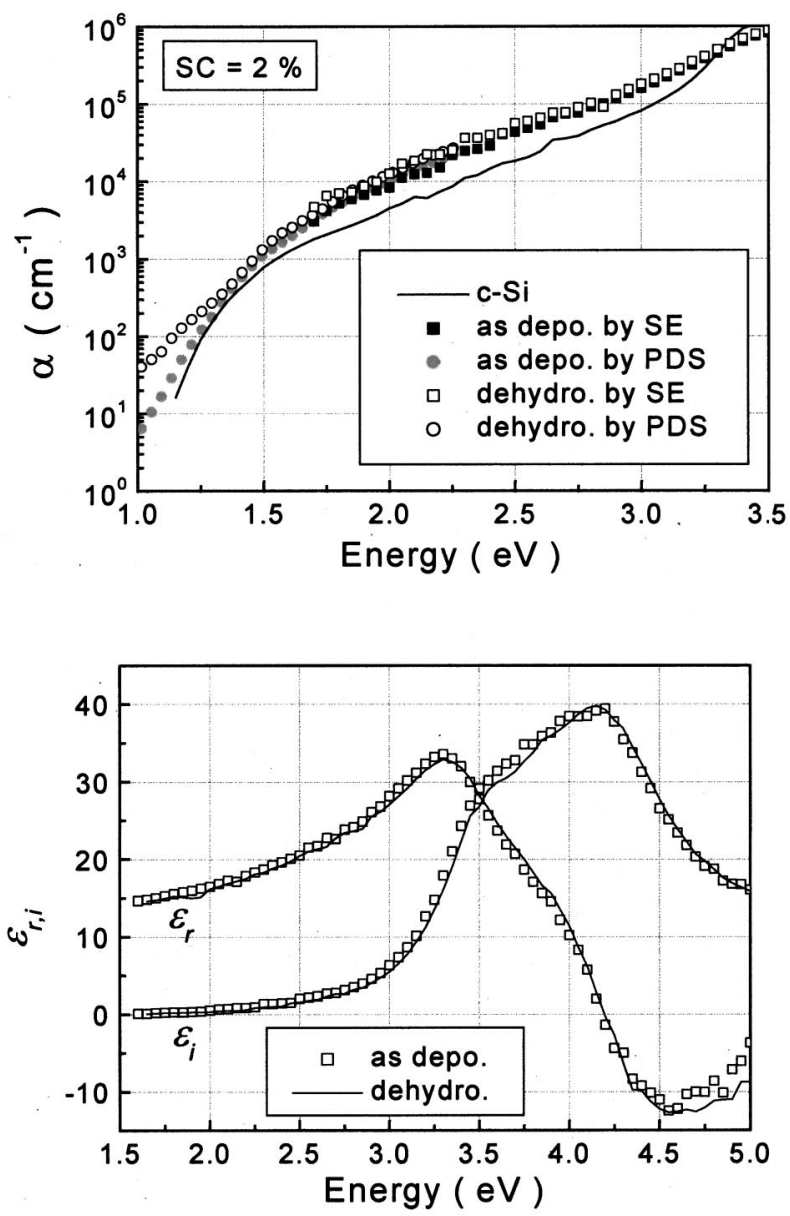

FIG. 8. $\alpha$ and $\left(\varepsilon_{r}, \varepsilon_{i}\right)$ of the $\mathrm{SC}=2 \% \mu c-\mathrm{Si}: \mathrm{H}$ in the asdeposited and the dehydrogenated. $\alpha$ was determined from SE and PDS, and $\left(\varepsilon_{r}, \varepsilon_{i}\right)$ was determined from SE. Some data points scatter due to the numerical instability. We truncated some largely deviating data points in $\alpha$ at the high-energy part of PDS and the low-energy part of SE. $\alpha$ of $c-\mathrm{Si}$ is shown as a reference.

duce $\alpha$ from the value of $c$-Si. Especially $\alpha$ of $\mathrm{SC}=2 \%$ $\mu c-\mathrm{Si}: \mathrm{H}$ is even higher than $c$-Si.

\section{A. Scattering effects as a reason for higher absorption for energies between 1.6 and $3.2 \mathrm{eV}$}

To explain the effect described in item (5b), we incorporate the scattering loss into the absorption coefficient calculated by Bruggeman-type EMT. Under independent scattering we have Rayleigh scattering coefficient for particles of radius $a$,

$$
\alpha_{s}=2 f_{s}\left(\frac{2 \pi}{\lambda} n_{s}\right)^{4} a^{3}\left|\frac{\varepsilon_{s}-\varepsilon_{1}}{\varepsilon_{s}+2 \varepsilon_{1}}\right|^{2},
$$

where $f_{s}$ is the volume fraction of the scatterers, $n_{s}$ is the real part of refractive index of the scatterers $\left(N_{s}=n_{s}+i k_{s}\right.$ $\left.=\sqrt{\varepsilon_{s}}\right), \varepsilon_{s}$ is the dielectric function of scatterer, $\varepsilon_{1}$ is the dielectric function of the matrix around the scatterers, and $\lambda$ is the wavelength of light in vacuum. We choose crystallites as the scatterer and amorphous matrix as the other part. Effective absorption coefficient is obtained by 


$$
\alpha_{e}=\alpha_{\mathrm{EMT}}+\alpha_{s}
$$

where $\alpha_{\mathrm{EMT}}$ is the absorption coefficient calculated by EMT. The effective refractive index is also changed correspondingly. We admit that this simple approach can only give a rough approximate estimation. To consider the multiple scattering effect and the accompanying depolarization effect, we employ the dense medium radiative transfer equation. A fully polarimetric description of the intensity is provided by the Stokes vector,

$$
\mathbf{I}(\theta, \phi, z)=\left[\begin{array}{c}
I_{p} \\
I_{s} \\
U \\
V
\end{array}\right]=\left[\begin{array}{c}
\left\langle E_{x} E_{x}^{*}\right\rangle \\
\left\langle E_{y} E_{y}^{*}\right\rangle \\
2\left\langle\operatorname{Re}\left(E_{x} E_{y}^{*}\right)\right\rangle \\
2\left\langle\operatorname{Im}\left(E_{x} E_{y}^{*}\right)\right\rangle
\end{array}\right]
$$

We consider the light incident from the air (Region $0, z>0$ ), reflected by a thin slab of $\mu c-\mathrm{Si}: \mathrm{H}$ (Region $1,-d<z<0$ ), and transmitted to the glass half plane (Region $2, z<-d$ ). Therefore, the incident beam in region 0 assumes the form

$$
\mathbf{I}_{0 i}\left(\pi-\theta_{0}, \quad \phi_{0}\right)=\left[\begin{array}{l}
1 \\
1 \\
2 \\
0
\end{array}\right] \delta\left(\cos \theta_{0}-\cos \theta_{0 i}\right) \delta\left(\phi_{0}\right),
$$

where the use of Dirac $\delta$ function is made. We chose the parameters (plane wave with amplitude 1 for each polarization and incident angle with $70^{\circ}$ as in conventional SE) to simulate the depolarization effect in the specular reflection direction $\left(\theta_{0}=\theta_{0 i}\right.$ and $\left.\phi_{0}=0^{\circ}\right)$. The dense medium radiative transfer equation that governs the propagation of intensities inside the $\mu c-\mathrm{Si}: \mathrm{H}$ slab are, for $0<\theta<\pi$,

$$
\begin{aligned}
\cos \theta \frac{d}{d z} \mathbf{I}(z, \theta, \phi)= & -\alpha_{e} \mathbf{I}(z, \theta, \phi)+\int_{0}^{\pi} d \theta^{\prime} \sin \theta^{\prime} \\
& \times \int_{0}^{2 \pi} d \phi^{\prime} \mathbf{P}\left(\theta, \phi ; \theta^{\prime}, \phi^{\prime}\right) \mathbf{I}(z, \theta, \phi),
\end{aligned}
$$

where $\mathbf{P}\left(\theta, \phi ; \theta^{\prime}, \phi^{\prime}\right)$ is a $4 \times 4$ scattering function matrix, which is identical to the Rayleigh phase matrix ${ }^{14}$ relating scattered intensities in the direction $(\theta, \phi)$ from incident intensities in the direction $\left(\theta^{\prime}, \phi^{\prime}\right)$. To satisfy the Rayleigh limit, the wavelength should be significantly larger than the size of the scatters, that is, $\left|(2 \pi / \lambda) N_{s} a\right| \ll 1$. The real angles of reflection and transmission at the boundary between the regions obey Snell's law by using the real part of the effective refractive index $(N=n+i k)$. Hence $n_{0} \sin \theta_{0}=n_{1} \sin \theta_{1}$ $=n_{2} \sin \theta_{2}$, where subscript denotes the region. We have to convert the real angle to a complex value to obtain Fresnel reflection and transmission coefficients at the boundaries. The relation between the real and complex angle is $N \sin \widetilde{\theta}$ $=n \sin \theta$ and $N \cos \widetilde{\theta}=n \cos \theta+i k$. The boundary conditions are, for $0<\theta<\pi / 2$, at $z=0$,

$$
\begin{aligned}
I(\pi & -\theta, \phi, z=0) \\
& =\overline{\overline{\mathbf{T}}}_{01}\left(\theta_{0}\right) \mathbf{I}_{o i}\left(\pi-\theta_{0}, \phi_{0}\right)+\overline{\overline{\mathbf{R}}}_{01}(\widetilde{\theta}) \mathbf{I}(\theta, \phi, z=0)
\end{aligned}
$$

and, at $z=-d$,

$$
\mathbf{I}(\theta, \phi, z=-d)=\overline{\overline{\mathbf{R}}}_{12}(\widetilde{\theta}) \mathbf{I}(\pi-\theta, \phi, z=-d),
$$

where $\mathbf{I}(\pi-\theta, \phi, z)$ and $\mathbf{I}(\theta, \phi, z)$ represent downwardgoing intensities and upward-going intensities, respectively, in region 1 . We used the complex angle $(\widetilde{\theta})$ for reflection and transmission coefficients. This interpretation of boundary condition may be the major difference from the dense medium radiative transfer theory for radiometry. We adopted the reflectivity and transmittivity matrices for plane waves $\overline{\overline{\mathbf{T}}}_{01}\left(\theta_{0}\right), \overline{\overline{\mathbf{R}}}_{01}(\widetilde{\theta})$, and $\overline{\overline{\mathbf{R}}}_{12}(\widetilde{\theta})$ as in Ref. 33 .

Conventionally solving the radiative transfer equation, the total scattered intensity is first decomposed into reduced intensity and diffused intensity. ${ }^{15}$ The reduced intensity can be obtained by solving the radiative transfer equation in the absence of scattering. For the calculation of the coherent Stokes vector, we took wave approach to calculate the reflectivity (direct reflection from the surface + transmission of reduced intensity to region 0 ) by using multiple-reflection and transmission equations with the effective refractive index,

$$
R_{p, s}=\left|\frac{r_{01}+r_{12} e^{(4 \pi / \lambda) N d}}{1+r_{01} r_{12} e^{(4 \pi / \lambda) N d}}\right|^{2},
$$

where $r_{a b}$ is the Fresnel reflection coefficient of $p$ mode or $s$ mode at the boundary $a$ and $b, N$ is relative value of refractive index of the slab. We can construct the coherent response of the Stokes vector from Eq. (10).

$$
\begin{aligned}
& \mathbf{I}_{0, \text { coherent }}\left(\theta_{0}=\theta_{0 i}, \phi_{0}=0^{\circ}\right) \\
& \quad=\left[\begin{array}{c}
\left|R_{p}\right|^{2} \\
\left|R_{s}\right|^{2} \\
2 \operatorname{Re}\left\langle R_{p} R_{s}^{*}\right\rangle \\
2 \operatorname{Im}\left\langle R_{p} R_{s}^{*}\right\rangle
\end{array}\right] \delta\left(\cos \theta_{0}-\cos \theta_{0 i}\right) \delta\left(\phi_{0}\right) .
\end{aligned}
$$

For the calculation of the incoherent Stokes vector, we rewrite the equation to calculate the diffused intensity as, for $0<\theta<\pi$

$$
\begin{aligned}
\cos \theta \frac{d}{d z} \mathbf{I}_{d}(\theta, \phi, z)= & -\alpha_{e} \mathbf{I}_{d}(\theta, \phi, z)+\int_{0}^{\pi} d \theta^{\prime} \sin \theta^{\prime} \\
& \times \int_{0}^{2 \pi} d \phi^{\prime} \mathbf{P}\left(\theta, \phi ; \theta^{\prime}, \phi^{\prime}\right) \mathbf{I}_{d}(\theta, \phi, z) \\
& +\int_{0}^{\pi} d \theta^{\prime} \sin \theta^{\prime} \int_{0}^{2 \pi} d \phi^{\prime} \mathbf{P}\left(\theta, \phi ; \theta^{\prime}, \phi^{\prime}\right) \\
& \times \mathbf{I}_{r}(\theta, \phi, z),
\end{aligned}
$$




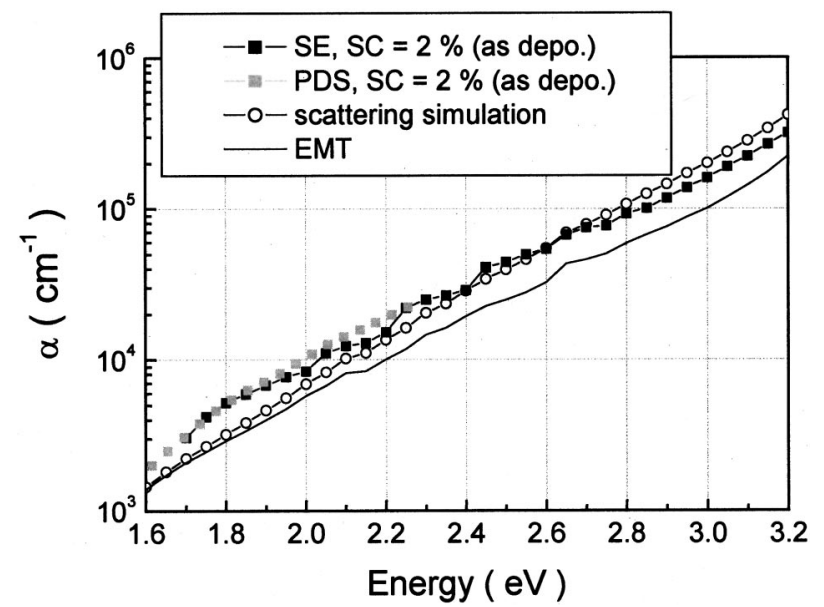

FIG. 9. $\alpha$ of $\mathrm{SC}=2 \% \mu c$-Si:H measured by SE and PDS, and $\alpha$ of $\mu c-\mathrm{Si}: \mathrm{H}$ from the Bruggeman-type EMT and considering Rayleigh scattering. Volume fraction of crystallite scatterer $f_{c}$ is 0.92 , volume fraction of surrounding $a-\mathrm{Si}: \mathrm{H}$ matrix $f_{a}$ is 0.05 , volume fraction of void $f_{v}$ is 0.03 , and the radius $(a)$ of the crystallite is 17 nm.

where $\mathbf{I}_{d}(\theta, \phi, z)$ denotes diffused intensity and $\mathbf{I}_{r}(\theta, \phi, z)$ denotes reduced intensity inside the random medium. For 0 $<\theta<\pi / 2$,

$$
\begin{aligned}
\mathbf{I}_{r}(\theta, \phi, z)= & \exp \left(-\alpha_{e} z / \cos \theta_{1}\right)\left[\mathbf{U}-\overline{\overline{\mathbf{R}}}_{10}\left(\widetilde{\theta}_{1}\right)\right. \\
& \left.\cdot \overline{\overline{\mathbf{R}}}_{12}\left(\widetilde{\theta}_{1}\right) \exp \left(-2 \alpha_{e} d / \cos \theta_{1}\right)\right]^{-1} \\
& \times \exp \left(-\alpha_{e} d / \cos \theta_{1}\right) \overline{\overline{\mathbf{R}}}_{12}\left(\widetilde{\theta}_{1}\right) \cdot \overline{\overline{\mathbf{T}}}_{10}\left(\theta_{0}\right) \\
& \cdot \mathbf{I}_{0 i}\left(\pi-\theta_{0}, \phi_{0}\right)
\end{aligned}
$$

and

$$
\begin{aligned}
\mathbf{I}_{r}(\theta, \phi, z)= & \exp \left(+\alpha_{e}, z / \cos \theta_{1}\right)\left[\mathbf{U}-\overline{\overline{\mathbf{R}}}_{10}\left(\widetilde{\theta}_{1}\right)\right. \\
& \left.\cdot \overline{\mathbf{R}}_{12}\left(\widetilde{\theta}_{1}\right) \exp \left(-2 \alpha_{e} d / \cos \theta_{1}\right)\right]^{-1} \\
& \times \overline{\overline{\mathbf{T}}}_{10}\left(\theta_{0}\right) \cdot \mathbf{I}_{0 i}\left(\pi-\phi_{0}, \phi_{0}\right),
\end{aligned}
$$

where $\theta_{1}$ (or $\widetilde{\theta}_{1}$ for boundary condition) is the angle inside the material corresponding to the incident angle by Snell's law.

The Fourier series expansions in azimuthal direction are applied to the Stokes vector and scattering matrix in order to eliminate the $\phi$ dependence. The Fourier series expansion of the Rayleigh scattering matrix and incident vector is given in Refs. 14 and 15. We solve the integro-differential equation using the Gaussian quadrature method. ${ }^{33,34}$ The consequent systems of first-order differential equations are then solved by obtaining eigenvalues and eigenvectors and matching the boundary conditions. ${ }^{14,15}$

In this paper, we simulate the incoherent Stokes vector scattered to the direction of specular reflection by

$$
\begin{aligned}
\mathbf{I}_{0, \text { incoherent }}\left(\theta_{0}\right. & \left.=\theta_{0 i}, \phi=0^{\circ}\right) \\
& =\overline{\overline{\mathbf{T}}}_{10}\left(\widetilde{\theta}_{1}\right) \mathbf{I}_{d}\left(\theta=\theta_{1}, \phi=0^{\circ}, z=0\right) .
\end{aligned}
$$

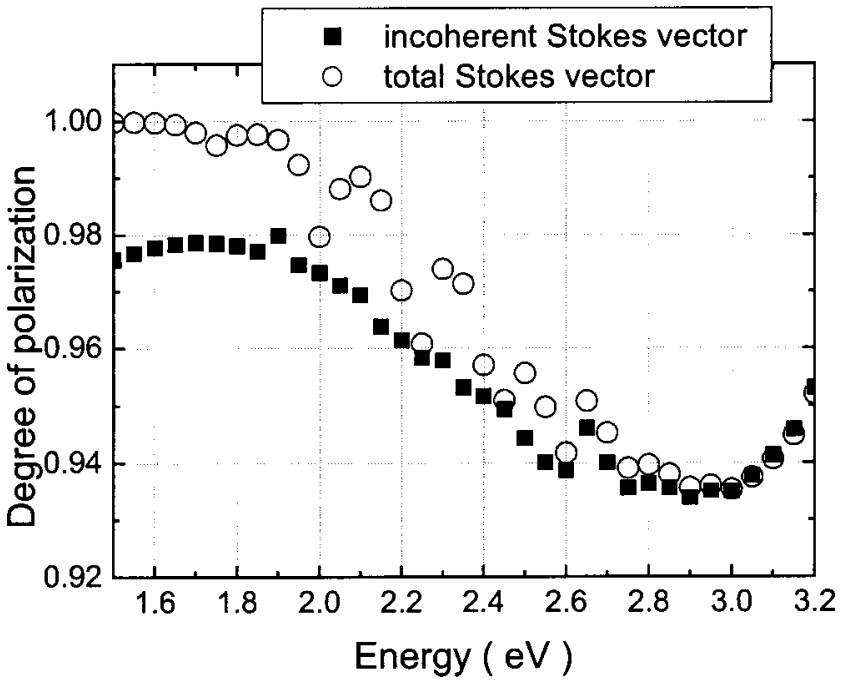

FIG. 10. Degree of polarization of incoherent and total Stokes vectors in the specular reflection direction. The same parameters as in Fig. 9 were used. The structure is $p$ - and $s$-polarized plane wave with unit intensity impinge from air to $\mu c-\mathrm{Si}: \mathrm{H}$ slab/glass half plane. The thickness of $\mu c-\mathrm{Si}: \mathrm{H}$ slab $(d)$ is $500 \mathrm{~nm}$.

By adding the coherent and incoherent Stokes vectors, we get total Stokes vector at $\theta=\theta_{0 i}$ direction. To demonstrate the multiple scattering effect, we calculate the degree of polarization defined as

$$
\mathrm{d}_{\mathrm{pol}}=\frac{\sqrt{\left(I_{p}-I_{s}\right)^{2}+U^{2}+V^{2}}}{I_{p}+I_{s}} .
$$

Figure 9 shows $\alpha$ of $\mathrm{SC}=2 \% \mu c-\mathrm{Si}: \mathrm{H}$ measured by $\mathrm{SE}$ and PDS, and the calculated $\alpha$ of $\mu c-$ Si:H with $f_{c}=0.92, f_{a}$ $=0.05$, and $f_{v}=0.03$. Here, $f_{a}$ denotes the amorphous volume fraction and $f_{v}$ denotes the void volume fraction, from the scattering simulation and from the Bruggeman-type EMT. We can see the impact of Rayligh scattering on the effective calculated absorption coefficient. In Fig. 9, the absorption coefficient from Eqs. (3) and (4) tends to be overestimated as the energy becomes higher and underestimated as the energy becomes lower. To be more quantitative, we have to consider (i) a new scattering model beyond the Rayleigh limit and (ii) clustering effect ${ }^{16}$ of the scatterers. The Mie scattering formalism can give more realistic results in the higher energy range. In the lower energy range, the scattering effect by crystallites are expected to disappear as the wavelength becomes larger. Thus we would attribute the distinct enhancement of $\alpha$ to strain effect in the grain and grain boundary. This will be discussed later.

The major advantage of this scattering simulation is that we can obtain all the four Stokes parameters, where depolarization effect can be assessed. Figure 10 shows the degree of polarization of incoherent and total Stokes vectors in the specular reflection direction when unit plane waves impinged. The parameters of the simulation are the same as used for the calculation for the curves in Fig. 9. This quantity cannot be calculated by the conventional wave approach. We can find that the depolarization effect diminishes as the photon energy approaches lower energy $(1.6 \mathrm{eV})$. 


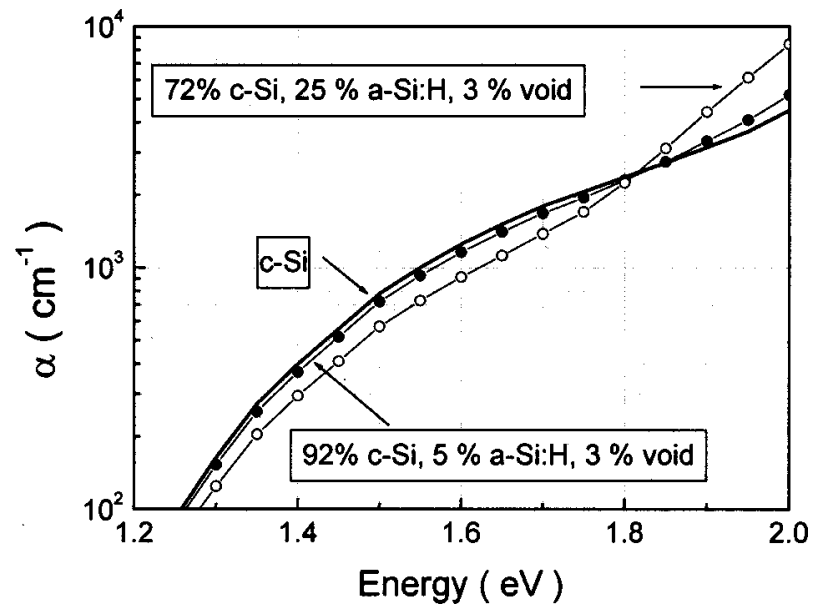

FIG. 11. $\alpha$ of $\mu c$-Si:H calculated by Bruggeman-type EMT. For the amorphous component, a device quality $a-\mathrm{Si}: \mathrm{H}$ film was deposited and measured by the authors. It should be noted that we used the bulk $c$-Si quantity for the calculation of crystalline phase.

\section{B. Strain as a reason for higher absorption for energies between 1.2 and $1.8 \mathrm{eV}$}

As we have shown in Figs. 9 and 10, another $\alpha$-enhancing mechanism has to be incorporated in the lower energy range (5c). Since the scattering effect has no significant influence on $\alpha$ in this energy range, we show in Fig. $11 \alpha$ of $\mu c-\mathrm{Si}: \mathrm{H}$ calculated by Bruggeman-type EMT using ideal composites. We chose the bulk $c$-Si value for crystallites and chose the measured value for $a-\mathrm{Si}: \mathrm{H}$ which shows a high performance in an active layer for solar cells. We show $\alpha$ for two different $f_{c} \mu c$-Si:H since we believe that the true $f_{c}$ of the compared $\mu c$-Si:H must lie between the two values. In the energy range of $E<1.8 \mathrm{eV}$, the absorption of $c$-Si is an upper limit for the absorption in the crystalline phase. By incorporating several percentage of $a-\mathrm{Si}: \mathrm{H}$ and voids in $c$-Si, a reduction in optical absorption can be observed which is caused by the low absorption of $a$-Si:H (e.g., $\alpha=47 \mathrm{~cm}^{-1}$ for $E=1.4 \mathrm{eV}$ ) and $\alpha=0 \mathrm{~cm}^{-1}$ for voids. $\alpha$ at $10^{3} \mathrm{~cm}^{-1}$ is shifted of around 0.08 and $0.13 \mathrm{eV}$ to higher energies for the calculated curves with $f_{c}$ of $92 \%$ and $72 \%$, respectively, in comparison with $\mathrm{SC}=2 \% \mu c-\mathrm{Si}: \mathrm{H}$ in Fig. 8. The amount of the energy shift represents how much the $\mathrm{SC}=2 \% \mu c-\mathrm{Si}: \mathrm{H}$ is in contradiction to the theoretical prediction by the ideal composites. This result is based on the assumption of the optical properties for bulk $c$-Si for the calculation of the crystalline phase. A loss of long-range periodicity in the crystalline phase causes a relaxation of the selection rules for optical transitions in the indirect transition regime where the momentum conservation is essential, however, it is in contradiction to the experimental data at lower photon energy $(E<1.8 \mathrm{eV})$, which shows more indirectlike transition.

The variation of the band tails can be interpreted in terms of localized states associated with the disorder that would be induced by a disordered phase. Werner et al. mentioned that electronic properties of poly-Si films are primarily determined by potential fluctuations. ${ }^{35}$ Fluctuations in the bond length and angle distribution around grain boundaries inevitably first lead to potential fluctuations and then give rise to localized tail states. Han et al. reported the result of photoluminescence (PL) spectra of the $\mu c-\mathrm{Si}: \mathrm{H}$ deposited by hotwire chemical-vapor deposition at different SC. ${ }^{36}$ As we can see from Ref. 36, redshift of the PL peak position $(1.0<E$ $<0.84 \mathrm{eV})$ is the dominant factor when an obvious $f_{c}$ emerges. They also attributed this effect to the ordering of the lattice and bond length, that is, strain effect. We account for $(5 c)$ by different amount of strain in each $\mu c-\mathrm{Si}: \mathrm{H}$. The similarity in $\alpha$ for $1<E<1.5 \mathrm{eV}$ for the $\mathrm{SC}=2 \%$ and $4 \%$ samples after dehydrogenation imply that $\mathrm{Si}-\mathrm{Si}$ network (without $\mathrm{H}$ ) is similar in both samples. We conclude that strain is mostly tensilelike in high- $f_{c} \mu c-\mathrm{Si}: \mathrm{H}$ by viewing the enhancement of $\alpha$. We introduce Volmer-Weber growth in poly-Si (or high- $f_{c} \mu c-\mathrm{Si}: \mathrm{H}$ ) formation. ${ }^{37-39}$ Tensile strain is generated by a Volmer-Weber mechanism. As growing crystallites contact each other at their bases, the sidewalls "zipped" together until a balance is reached between the energy associated with eliminating surface area, creating a grain boundary and straining the film. In this process, strain depends on interfacial free energies, elastic properties, and grain size. As two islands impinge and form a grain boundary at their intersection, sidewall of the free surface of each island is eliminated, resulting in a significant energy reduction. The second source of tensile strain is micropores that induce an elastic strain in the anchored grains due to a constrained relaxation. The third source of tensile strain is removal of $\mathrm{H}$ around the $c$-Si grains, either by extensive chemical annealing or thermal annealing, in other words, surface reconstruction arises after removal of $\mathrm{H}$ bonds. $\mathrm{H}$-induced strain may build up due to trapped $\mathrm{H}_{2}$, otherwise, $\mathrm{H}$ incorporation need not contribute to intrinsic stress since its incorporation occurs integrally with the formation of the rigid silicon network at the growing surface. On the contrary, if $\mathrm{H}$ incorporation is decreased with decreasing SC (see Fig. 5), elimination of strained $\mathrm{Si}-\mathrm{Si}$ bonds due to in-diffusion process can be decreased. In support of this, studies of glow discharge deposition conditions include a demonstration that the onset of columnar growth morphology is accompanied by an abrupt drop in compressive stress. ${ }^{40}$ In porous silicon, the enhancement of $\left(\varepsilon_{r}, \varepsilon_{i}\right)$ was attributed to the change in the level of strain present in the cellular structure of the porous silicon film and which is induced by $\mathrm{H}$ desorption from the porous silicon film. ${ }^{41}$ Recrystallization studies by annealing of $a$-Si:H have demonstrated that as the $\mathrm{H}$ concentration decreases with increasing the anneal temperature, the initially compressive strain changes to a tensile strain. ${ }^{42} \mathrm{Be}-$ side the higher absorption also the electronic properties are affect by strain. Solar cells from bulk $c$-Si have shown $\mathrm{V}_{\mathrm{oc}}$ $\sim 0.65 \mathrm{~V} .{ }^{43}$ On the other hand, the solar cell incorporating intrinsic $\mu c-\mathrm{Si}: \mathrm{H}$ as an active layer has shown $\mathrm{V}_{\mathrm{oc}} \sim 0.39 \mathrm{~V}$ at $\mathrm{SC}=2 \%$ and $\mathrm{V}_{\mathrm{oc}} \sim 0.55 \mathrm{~V}$ at $\mathrm{SC}=5 \%$, where the latter condition shows an optimum performance. ${ }^{1,2}$ The lower $\mathrm{V}_{\mathrm{oc}}$ of the $\mu c-\mathrm{Si}: \mathrm{H}$ solar cells has been usually attributed to the poor junction properties-high roughness and profile inhomogeneity of the $\mu c-\mathrm{Si}: \mathrm{H}$ active layer said to deteriorate the interface quality of $p-i-n$ junction. This paper suggests that the tensile strain in the crystalline phase of $\mu c-\mathrm{Si}: \mathrm{H}$ can be a factor lowering $\mathrm{V}_{\mathrm{oc}}$ of $\mu c-\mathrm{Si}: \mathrm{H}$ solar cells. 


\section{SUMMARY}

We have investigated the optical properties of intrinsic microcrystalline silicon $(\mu c-\mathrm{Si}: \mathrm{H})$ prepared at different $\mathrm{SC}$ $(2 \%<\mathrm{SC}<7 \%)$ by SE and PDS for photon energies between 1.0 and $5.0 \mathrm{eV}$. Mechanical polishing improves the evaluation of the bulk properties of the microcrystalline material significantly and reduce the impact of surface layers. At high SC, we extracted the optical characteristics of the disordered part in a limited energy range of $1.5<E$ $<3.2 \mathrm{eV}$ by assuming a superposition of the optical properties of ideal $c$-Si and the disordered part. At low SC, we paid special attention to the energy range at $1.6<E<3.2 \mathrm{eV}$, where the small amorphous volume in a highly crystalline $\mu c-\mathrm{Si}: \mathrm{H}$ cannot account for the high absorption of light. We explained the enhanced $\alpha$ in the energy range by Rayleigh scattering and simulated the multiple scattering effect through dense medium radiative transfer equation. We took wave approach in calculating the coherent Stokes vector in the specular reflection direction and converted the real angle to the complex value in calculating the transmission and reflection coefficients. We found that the optical scattering effect brings about depolarization of $s$ and $p$ waves while generating incoherent waves. We also suggested strain effect as a possible reason for the enhanced absorption in the highly crystalline $\mu c-\mathrm{Si}: \mathrm{H}$ by viewing the onset of the indirect transition regime $(1.2<E<1.8 \mathrm{eV})$. We discussed the sources for strain in $\mu c-\mathrm{Si}: \mathrm{H}$.

\section{ACKNOWLEDGMENTS}

The authors would like to thank H. J. Eom, W. Beyer, and F. Finger for helpful discussions, A. Lambertz for sample preparation, and J. Klomfass for PDS measurements and sample annealing.
${ }^{1}$ O. Vetterl, F. Finger, R. Carius, P. Hapke, L. Houben, O. Kluth, A. Lambertz, A. Mück, B. Rech, and H. Wagner, Sol. Energy Mater. Sol. Cells 62, 97 (2000).

${ }^{2}$ A. Shah, E. Vallat-Sauvain, P. Torres, J. Meier, U. Kroll, C. Hof, C. Droz, M. Goerlitzer, N. Wyrsch, and M. Vaněček, Mater. Sci. Eng., B 69-70, 219 (2000).

${ }^{3}$ N. Layadi, P. Roca i Cabarrocas, B. Drévillon, and I. Solomon, Phys. Rev. B 52, 5136 (1995).

${ }^{4}$ P. Roca i Cabarrocas, N. Layadi, T. Heitz, and B. Drévillon, and I. Solomon, Appl. Phys. Lett. 66, 3609 (1995).

${ }^{5}$ P. Petrik, T. Lohner, M. Fried, L. P. Biró, N. Q. Khánh, J. Gyulai, W. Lehnert, Schneider, and Ryssel, J. Appl. Phys. 87, 1734 (2000).

${ }^{6}$ J. Ferber and J. Luther, Sol. Energy Mater. Sol. Cells 54, 265 (1998).

${ }^{7}$ G. Rothenberger, P. Comte, and M. Grätzel, Sol. Energy Mater. Sol. Cells 58, 321 (1999).

${ }^{8}$ A. Achiq, R. Rizk, F. Gourbilleau, R. Madelon, B. Garrido, A. Pérz-Rodríguez, and J. R. Morante, J. Appl. Phys. 83, 5797 (1998).

${ }^{9}$ J.C. Knight, 1984 in Topics in Applied Physics-Hydrogenated Amorphous Silicon, edited by J.D. Joannopoulos, G. Lucovsky, (Springer Verlag, Heidelberg, 1984), Vol. 55, p. 5.

${ }^{10}$ G. E. Jellison, Jr., M. F. Chisholm, and S. M. Gorbatkin, Appl. Phys. Lett. 62, 3348 (1993).

${ }^{11}$ G. E. Jellison, Jr., S. P. Withrow, J. W. McCamy, J. D. Budai, D. Lubben, and M. J. Godbole, Phys. Rev. B 52, 14607 (1995).

${ }^{12}$ L. Viña and M. Cardona, Phys. Rev. B 29, 6739 (1984).

${ }^{13}$ N. Beck, J. Meier, J. Fric, Z. Remeš, A. Poruba, R. Flueckiger, J. Pohl, A. Shah, and A. Vaněček, J. Non-Cryst. Solids 198-200, 903 (1996).

${ }^{14}$ R. T. Shin and J. A. Kong, J. Appl. Phys. 52, 4221 (1981).

${ }^{15} \mathrm{~B}$. Wen, L. Tsang, D. P. Winebrenner, and A. Ishimaru, IEEE Trans. Geosci. Remote Sens. 28, 46 (1990).

${ }^{16}$ L. Tsang and J. A. Kong, in Scattering of Electromagnetic Waves Vol. I and III (Wiley, New York, 2001)

${ }^{17}$ W. Beyer, J. Herion, H. Wagner, and U. Zastrow, Philos. Mag. B 63, 269 (1991).
${ }^{18}$ W. Beyer, Physica B 170, 105 (1991).

${ }^{19}$ W. Jackson, N. M. Amer, A. C. Boccara, and D. Fournier, Appl. Opt. 20, 1333 (1981).

${ }^{20}$ N. M. Amer and W. B. Jackson, in Semiconductors and Semimetals, edited by J. J. Pankove (Academic, New York, 1984), Vol. 21B, p. 83.

${ }^{21}$ D. Ritter, K. Weiser, Opt. Comm. 57, 336 (1986).

${ }^{22} \mathrm{R}$. Benferhat, in Optical Characterization of thin film (JobinYvon, France, 1996), p. 49.

${ }^{23}$ D. A. G. Bruggeman, Ann. Phys. (Leipzig) 24, 636 (1935).

${ }^{24}$ G. E. Jellison, Jr. and F. A. Modine, Appl. Phys. Lett. 69, 371 (1996).

${ }^{25}$ L. Houben, M. Luysberg, P. Hapke, R. Carius, F. Finger, and H. Wagner, Philos. Mag. A 77, 1447 (1998); M. Luysberg, C. Scholten, L. Houben, R. Carius, F. Finger, O. Vetterl, Mat. Res. Soc. Symp. Proc. 664 (2001), p. A15.2.1.

${ }^{26}$ T. D. Moutakas, D. A. Anderson, and W. Paul, Solid State Commun. 23, 43 (1980).

${ }^{27}$ C. C. Tsai, in Amorphous Silicon and Related Materials (Ref. 21), p. 123.

${ }^{28}$ H. V. Nguyen, Y. Lu, S. Kim, M. Wakagi, and R. W. Collins, Phys. Rev. Lett. 74, 3880 (1995).

${ }^{29}$ K. H. Jun, K. S. Lim, S. Y. Kim, and S. J. Kim, J. Non-Cryst. Solids 275, 59 (2000).

${ }^{30}$ B. Johs, in Software for VASE, version 2.46 (Wollam, Lincoln, NE, 1995).

${ }^{31}$ S. Adachi and H. Mori, Phys. Rev. B 62, 10158 (2000).

${ }^{32}$ A. Borghesi, M. E. Giardini, M. Marazzi, A. Sassella, and G. De Santi, Appl. Phys. Lett. 70, 892 (1997).

${ }^{33}$ Chi M. Larn and A. Ishimaru, IEEE Trans. Antennas Propag. 41, 851 (1993)

${ }^{34} \mathrm{~S}$. Chandrasekhar, in Radiative Transfer (Dover, New York, 1960).

${ }^{35}$ J. Werner and M. Peisl, Phys. Rev. B 31, 6881 (1985).

${ }^{36}$ D. Han, G. Yue, J. D. Lorentzen, J. Lin, H. Habuchi, and Q. Wang, J. Appl. Phys. 87, 1882 (2000).

${ }^{37}$ W. D. Nix and B. M. Clemens, J. Mater. Res. 14, 3467 (1999). 
${ }^{38}$ S. C. Seel, C. V. Thompson, S. J. Hearne, and J. A. Floro, J. Appl. Phys. 88, 7079 (2000).

${ }^{39}$ J. A. Floro, S. J. Hearne, J. A. Hunter, P. Kotula, E. Chason, S. C. Seel, and C. V. Thompson, J. Appl. Phys. 89, 4886 (2001).

${ }^{40}$ J. P. Harbison, A. J. Williams, and D. V. Lang, J. Appl. Phys. 55, 946 (1984).
${ }^{41}$ A. Larré, A. Halimaoui, F. Glowacki, F. Ferrieu, Y. Campidelli, and D. Bensahel, Appl. Phys. Lett. 65, 1566 (1994).

${ }^{42}$ P. Paduschek, Ch. Höpfl, and H. Mitlehner, Thin Solid Films 110, 291 (1983).

${ }^{43}$ A. W. Blakers and M. A. Green, Appl. Phys. Lett. 48, 215 (1986). 